https://doi.org/10.31713/m1009

\title{
MODERNIZATION OF METALLURGICAL EQUIPMENT WITH THE DEVELOPMENT OF AN INNOVATIVE METHOD OF COILING HOT-ROLLED STRIPS
}

\author{
Kassym Yelemessov \\ Satbayev University, Candidate of Technical Sciences, Associate \\ Professor, Director of the Institute of Metallurgy and Industrial \\ Engineering, Republic of Kazakhstan
}

Kanay Rysbekov

Satbayev University, Candidate of Technical Sciences, Associate

Professor, Director of the Institute of Geology, Petroleum and

Mining engineering, Republic of Kazakhstan

\section{Dinara Baskanbayeva}

Satbayev University, Master of Technical Sciences, senior researcher, Republic of Kazakhstan

\section{Akzhan Igbayeva}

Satbayev University, Master of Technical Sciences, researcher, Republic of Kazakhstan

\section{Annotation (Abstract)}

The report presents the results of laboratory studies on the use of a combined (water and air) method of coiling hot-rolled strips on a coiler of a continuous broadstrip mill 1700. An innovative method of coiling strips allows to completely abandon the mechanical formation of hot-rolled coils. In order to study the hydraulic formation in laboratory conditions, a model of a coiler was created, which was structurally tied to the existing laboratory rolling mill "DUO-100". Studies carried out on the laboratory model of a coiler with hydraulic roll formation confirmed the fundamental possibility of using the proposed coiling method for working with various materials. Aluminum and lead strips were used as hot rolled strip. The results of laboratory work indicate a sufficient convergence of calculations with experimental data, which allows using the results obtained as recommendations for the creation of industrial installations. A method for calculating the flow rate and pressure of water and air is proposed. The calculation of the clamping force of the front end of the strip and the refined selection of the number of collectors and the maximum angle of rotation of the nozzle are carried out. The use of the combined principle for the formation of the first turns of the roll avoids contact between the rolled metal and the strip, expands the adjustment limits and reduces the requirements for its accuracy. The results obtained make it possible to recommend the use of a combined method of strip coiling in rolling production. 


\section{Introduction}

Steel sheet production is the backbone of ferrous metallurgy. More than $80 \%$ of steel smelted at metallurgical plants is used for the manufacture of rolled products: thick and thin sheets, pipes of various diameters, bent sections from sheet metal, etc. $[1,2]$. Widestrip hot rolling mills for steel strip have found wide application in the global production of rolled steel. The reason for this was the development of technologies for obtaining finished products from sheet material, such as stamping, bending of profiles, obtaining welded pipes of various sections and purposes.

Continuous wide-strip mill 1700 (CWSM) is designed for rolling steel strip with a thickness of $1.8 \mathrm{~mm}$ to $12 \mathrm{~mm}$ and a width of up to $1550 \mathrm{~mm}$ $[3,4]$.

One of the main conditions for increasing production efficiency is increasing reliability, reducing downtime, extending the overhaul periods of rolling equipment, starting from the loading device and ending with the lifting-rotary tables of the mill.

From the analysis of the content of works devoted to the coiling of wide hot-rolled strips, the following conclusion can be drawn. The works of the authors of the CIS and most of the works of authors from Western countries are aimed at changing the design as a whole, improving individual units, and sometimes devoted to the problems of technology. The vast majority of Japanese works are devoted to the automation of the process, the use of various sensors in conjunction with computer systems, and the use of algorithms developed by the authors in the process of winding [5-10].

In order to study the innovative system of coiling at the Kazakh National Research Technical University named after K.I. Satbayev (Satbayev University), the authors carried out special studies to create a laboratory installation for a combined method of coiling hot-rolled strips [11].

The following tasks were solved:

- creation of the laboratory design of the coiler with the combined strip coiling system;

- development of the method for calculating the clamping of the front end of the strip;

- calculation of consumption, pressure of water and air;

The results of these studies are presented in this report. 


\section{Methods}

During the research, a comprehensive methodology was used, including an analytical review of literary sources, a review of the operating experience and yield of suitable products while coiling of strips with coilers of industrial enterprises in Kazakhstan and foreign countries from reporting and statistical data. Theoretical studies of the technical parameters of a new design of coilers and experimental studies of coiling methods were also used.

This data will make it possible to evaluate and highlight the main influencing factors that will allow the creation of an innovative and efficient system for coiling hot-rolled strips. Experimental studies were carried out on a new design of coilers with reference to the laboratory rolling mill "DUO-100", and their results were processed by methods of mathematical statistics according to standard techniques. In the course of laboratory studies of the coiler model with the combined roll formation, one of the main parameters was the force from the side of a stream of water and air on the surface of the drum or on the coiled strip. The nozzles themselves used in the installation had calibrated holes, for which precisely manufactured jets from automobile carburetors were used.

Lead and aluminum strips were used as hot-rolled strip, which perfectly simulated the parameters of hot-rolled strips. This ensured a high reliability of the results obtained and made it possible to carry out calculations on the flow rate and pressure, as well as to develop a method for calculating the pressure of the front end of the strip.

\section{Results and discussion}

The development was based on a British patent [12], which proposed a device for winding thin metal strips (foil) by pressing the front end of the strip to the drum with a jet of compressed air. The air supply manifold bends around the reel drum in a circle and after the strip is captured, it can be removed from the surface of the coiled roll. The use of compressed air as a working medium limits the capabilities of this device, as the force from the side is jetted by its cross section, the speed of the working medium (air) outflow and the specific gravity of the working medium. It was proposed to form 
rolls of hot-rolled steel practice in a similar way, using water in the working body [11-14].

Initially, we have completely replaced the working body of the system, i.e. air to water and calculations were carried out only for the hydraulic system. However, after a series of discussions and preliminary publications, we received comments on the problem of water residual between the turns of the rolls. Subsequently, it was decided to use a combined method of strip coiling. Hot air under pressure completely removes water between the coils, thus ensuring high quality coiled strips.

\section{Coiler model design}

The model of the coiler is designed to study the parameters of winding on model materials, to work out various modes, conditions for forming a roll. To obtain information simulating the operation of coilers in the shop, the coiler model was structurally tied in its parameters to the existing laboratory model of the "Duo-100" rolling mill (Fig. 1,2).

Diagram of the laboratory rolling mill "Duo - 100" with a reel model

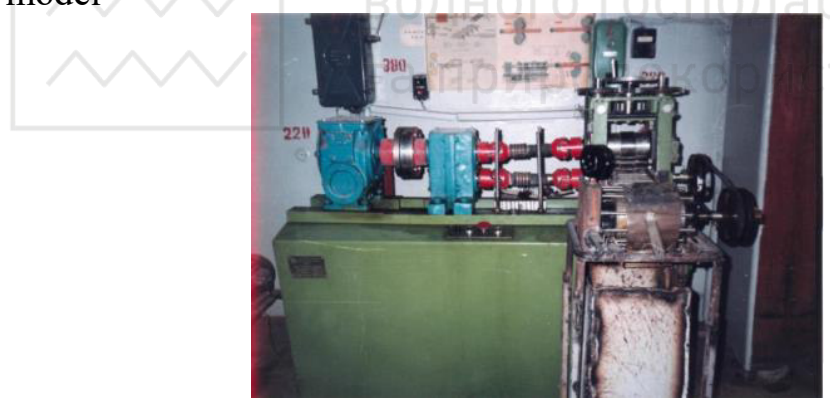

Fig. 1.

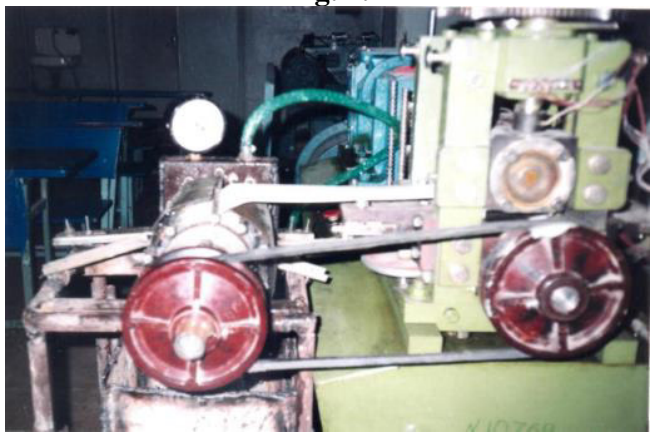

Fig. 2. 
The main unit of the coiler is a drum with a diameter of $300 \mathrm{~mm}$, made from a pipe. The length of the drum is $200 \mathrm{~mm}$, which corresponds to the length of the roll barrel of the rolling mill. The drum is connected through trunnions to the drive shaft mounted in roller bearings. In the above calculations, for the most difficult case of winding a lead strip, the required power of the electric motor is determined. However, in order to simplify the design and at the same time to expand the possibilities of adjusting the relative speeds of the work rolls of the mill and the drum, a $V$-belt transmission was used. Were used pulleys - variators, allowing you to change their radius by changing the distance between their cones. The driving pulley is fixed coaxially with the lower roll of the mill, and the driven pulley is fixed on the axis of the reel drum.

The hydraulic forming device is mounted independently of the drum on the non-drive side (Fig. 2). It is based on a sealed steel tank with a capacity of $0.002 \mathrm{~m}^{3}$ liters. It has two fittings for water supply and pressure gauge installation. On the side wall of the tank, perpendicular to the drum axis, collectors for supplying water and air to the winding strip are fixed.

The collectors are copper tubes with an outer diameter of $10 \mathrm{~mm}$ and an inner diameter of $8 \mathrm{~mm}$. One end of the tubes is plugged. In each manifold, five holes are drilled along one longitudinal line, designed for screwing calibrated nozzles into them. The pipe is not plugged at the other end and the thread is cut. With the help of the thread, the collector is attached to the wall of the water tank, where, in turn, the thread is cut. Such fastening allows, by turning the collectors in the thread, to change the angle of inclination of the nozzles relative to the drum surface. Holes for mounting collectors are located radially relative to the axis of the reel drum.

The diameter of the centers of the fixing holes determines the distance from the cut of the working nozzles to the surface of the drum and the coiled strip. The holes for screwing calibrated nozzles into the manifolds are arranged in such a way that they evenly overlap the entire width of the strip wound on the drum.

The bearing housings of the drum and the tank for supplying water to the manifolds are welded to a frame mounted on the floor. The height of the frame is such that the upper level of the drum is 5 $\mathrm{mm}$ above the pick-up table of the rolling mill and approximately at 
the upper level of the lower roll. The frame is equipped with waterproof casings that enclose the drum. The casings are made with a cut in the upper part, designed to feed the coiled strip from the rolling mill table to the drum. During the experiments, water is supplied to the reel model from the hydraulic pneumatic accumulators with a hose. The pressure in the reel tank is changed by adjusting the valve at the inlet to the tank according to the pressure gauge. Under the reel model there is a 40 liter water collection tank with a drain valve.

\section{Research of the winder model}

Calculations have shown the possibility of creating a laboratory design of a coiler designed for winding a lead strip $50 \mathrm{~mm}$ wide and up to $1 \mathrm{~mm}$ thick. In this case, the nozzle diameters of $0.7 \mathrm{~mm}, 0.9$ $\mathrm{mm}$ and $1.1 \mathrm{~mm}$ were chosen as the basis for the calculation.

However, when making the current working model, it was necessary to take into account the objective reasons that limit the capabilities of the device. Thus, a check with a pressure gauge in the water supply network showed that its maximum value does not exceed $0.38 \mathrm{MPa}$. The diameter of the outlet section of the valve for water supply is $12 \mathrm{~mm}$. Based on the specified pressure, we determine the force of the jet at nozzle diameters of $1 \mathrm{~mm}, 2 \mathrm{~mm}$ and $3 \mathrm{~mm}$.

For pneumatic and hydraulic systems, in order to ensure the amount of supplied liquid or gas at the operating pressure value, it is necessary that the total cross section of the jets at the final stage be less than or equal to the supply line. Otherwise, the volume or pressure of the supplied working fluid decreases. In the current model, copper tubes with an outer diameter of $10 \mathrm{~mm}$ and with an inner diameter of $8 \mathrm{~mm}$ were used as collectors for supplying water to the nozzles. The number of nozzles with a diameter of $1 \mathrm{~mm}$ can be no more than 144 pieces, with a diameter of $3 \mathrm{~mm}-16$ pieces, with a diameter of $5 \mathrm{~mm}-6$ pieces. Assuming that the number of supply manifolds must be at least six, and the number of nozzles on each manifold must be at least five, then the total minimum number of nozzles will be 30 pieces. 
Method for calculating the clamping force of the front end of the strip for a laboratory coiler

To determine the number of collectors (points of load application to the strip), we proceed from the following conditions:

1. The strip fed for coiling has the same width and thickness and is flat or nearly flat. This complies with the requirements for a rolled strip.

2. The task of the forming device is to provide compression of the strip to the surface of the drum along its entire generatrix (along the entire circumference). For the laboratory design of the coiler, it was decided to test the forming device without the use of additional mechanical guides.

The action of normal force to the surface of the drum, the movement of the front end of the strip can be viewed tangentially to this point.

For further winding, it is necessary that when moving, the front end must necessarily fall under the action of the next one. Consequently, the deformation of the surface of the coiled strip is less than $1 \%$. For a laboratory coiler, this ratio will be: $1 / 130=0.0077$. With such a small value of the total degree of deformation, the main part of the deformation will be elastic.

Since it is almost impossible to separate the plastic and elastic components of the deformation of the strip being coiled, then when determining the number of collectors, we assume that the deformation produced at the point of application of the force to the strip is mainly elastic. It follows from this that it is necessary to determine a sufficient number of points of application of force (jet), that is, it is necessary to choose the optimal number of collectors and the angles between them. To do this, we will use the graphic-analytical method and construct the intersection points of tangents and cuts of nozzles located at different distances from the drum surface.

Accordingly (Fig. 3), the number of nozzles decreases as they move away from the drum surface. The results are summarized in Table 1. 


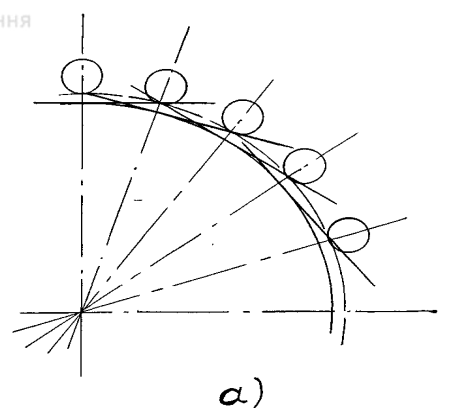

Fig. 3. Graphic-analytical method for determining the number of collectors for the coiler model

Table 1

The number of collectors and angles between them

\begin{tabular}{c|c|c|c|c|c}
\hline$h_{0}, \mathrm{~mm}$ & 3 & 5 & 10 & 15 & 20 \\
\hline $\cos \alpha$ & 0,942 & 0,915 & 0,855 & 0,802 & 0,756 \\
\hline$\alpha^{0}$ & 19,61 & 23,8 & 31,24 & 36,68 & 40,9 \\
\hline$\eta$ & 18,36 & 15,13 & 11,52 & 9,82 & 8,9 \\
\hline$\eta_{\phi}$ & 18 & 15 & 12 & 10 & 9 \\
\hline$\alpha_{\phi}^{0}$ & 20 & 24 & 30 & 36 & 40 \\
\hline
\end{tabular}

In order for the force from the side of the jet to have a pushing component, the jet must have an inclination in the direction of the movement of the strip. However, the angle of inclination cannot be greater than a certain value, since with its increase, simultaneously with the growth of the horizontal component, the normal one, which is responsible for pressing the strip to the drum and elastoplastic deformation, decreases.

The required force consists of two values - from the bending of the strip in the elastic-plastic region and from overcoming the centrifugal force. Figure 4 shows the direction of the forces acting on the strip to press it against the drum surface. 


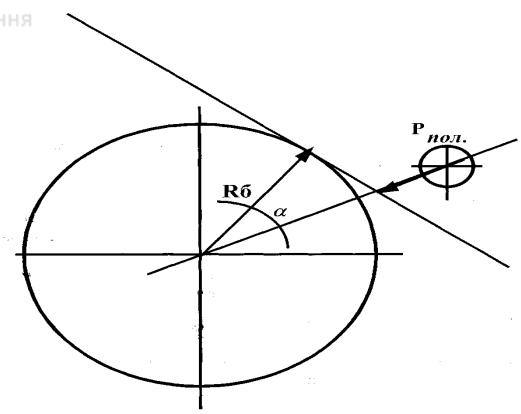

Fig. 4. The action of the forces forming the roll

The dimensions of the strips are taken constructively, based on the characteristics of rolling on a laboratory rolling mill:

width $b=50 \mathrm{~mm}=0.05 \mathrm{~m}$;

thickness $h=0.2 ; 0.5 ; 0.8 ; 1 \mathrm{~mm}=0.0002 ; 0.0005 ; 0.0008 ; 0.001$ $\mathrm{m}$;

$\alpha$ is the angle between forming rollers or nozzles (in our case, $\alpha=36^{\circ}$ ).

The total force acting on the front end of the strip will be made up of static and dynamic forces

$$
R_{\mathrm{pol}}=R_{\mathrm{st}}+R_{\mathrm{din}}
$$

The results of calculating the values of the clamping force are summarized in Table 2.

Table 2

The results of calculating the values of Pst, $\mathrm{N}$

\begin{tabular}{c|c|c|c|c}
\hline Strip width, $\mathrm{m}$ & 0,05 & 0,05 & 0,05 & 0,05 \\
\hline Strip thickness, $\mathrm{m}$ & 0,0002 & 0,0005 & 0,0008 & 0,001 \\
\hline Static force, $\mathrm{N}$ & 0,4 & 2,6 & 6,9 & 10,7 \\
\hline
\end{tabular}

The results of calculating the values of the mass and weight of one turn of the strip, as well as the dynamic force are shown in Table 3.

Table 3

The results of calculating the value of the mass and weight of one turn of the strip, as well as the dynamic force

\begin{tabular}{l|c|c|c|c}
\hline Thickness, m & 0,0002 & 0,0005 & 0,0008 & 0,001 \\
\hline Width, m & 0,05 & 0,05 & 0,05 & 0,05 \\
\hline Mass M, kg & 0,046 & 0,116 & 0,186 & 0,23 \\
\hline Weight W, N & 0,46 & 1,16 & 1,86 & 2,3 \\
\hline Dynamic force, N & 0,000185 & 0,00047 & 0,00075 & 0,00093 \\
\hline
\end{tabular}


From the Table 3 it follows that the dynamic forces to overcome the centrifugal force are insignificant due to the low rolling speed.

Based on the dimensions of the drum, the number of hydraulic supply lines is initially assumed to be ten with an even distribution across the diameter through $36^{\circ}$. The arrangement and the number of nozzles on each collector are determined from the condition of winding strips of different widths. All ten hydraulic feeding lines will operate in the winding of coils up to $50 \mathrm{~mm}$ wide.

The total force per nozzle system when winding strips of different thicknesses and widths is shown in Table 4.

Table 4

Total force $\mathrm{P}, \mathrm{N}$

\begin{tabular}{l|l|l|l|l}
\hline Strip width, $\mathrm{m}$ & 0,05 & 0,05 & 0,05 & 0,05 \\
\hline Strip thickness, $\mathrm{m}$ & 0,0002 & 0,0005 & 0,0008 & 0,001 \\
\hline Force, $\mathrm{N}$ & 0,4 & 2,6 & 6,9 & 10,7 \\
\hline
\end{tabular}

\section{Calculation of water pressure and flow}

Based on the arrangement of nozzles and the required values of the total forces on one hydraulic supply line (Table 4), dividing the latter by the number of nozzles in the line, we determine the required force of the water coming out of one nozzle. The calculated forces of the jet emerging from one nozzle are entered in Table 5.

Table 5

The force of the jet coming out of one nozzle, $\mathrm{N}$

\begin{tabular}{l|l|l|l|l}
\hline Strip width, $\mathrm{m}$ & 0,05 & 0,05 & 0,05 & 0,05 \\
\hline Strip thickness, $\mathrm{m}$ & 0,0002 & 0,0005 & 0,0008 & 0,001 \\
\hline Force, $\mathrm{N}$ & 0,08 & 0,52 & 1,38 & 2,14 \\
\hline
\end{tabular}

From Table 5 it follows that when bending strips with a width of $0.05 \mathrm{~m}$ and a thickness of $0.001 \mathrm{~m}$, the force of water should be equal to $2.14 \mathrm{~N}$. We take this value as the main one when carrying out further calculations. For the calculation, we set three nozzle diameters: $0.7 \mathrm{~mm}, 0.9 \mathrm{~mm}, 1.2 \mathrm{~mm}$.

The required force of the water jet on the strip is determined by the formula

$$
Q_{\mathrm{m}}=P \cdot V \cdot S,
$$

where $\rho$ is the density of water, $\rho=1000 \mathrm{~kg} / \mathrm{m}^{3}$;

$s$ - nozzle section, $\mathrm{m}^{2}$. 
We get the expression for the effort of the water jet

$$
P_{\mathrm{c}}=\rho \cdot s \cdot v^{2}
$$

Subsequent values of the speed of the water jet are given in Table 6 .

Table 6

Estimated value of water jet speed, $\mathrm{m} / \mathrm{s}$

\begin{tabular}{c|c|c|c|c|c}
\hline \multirow{2}{*}{ Section, $\mathrm{m}^{2}$} & \multirow{2}{*}{0,08} & \multirow{2}{*}{0,52} & \multicolumn{2}{|c|}{ Force, $\mathrm{N}$} & \multirow{2}{*}{} \\
\cline { 4 - 5 } & & & & 1,38 & \\
\hline $0,4 \cdot 10^{-6}$ & 14,14 & 36.1 & & 58,74 & $0,4 \cdot 10^{-6}$ \\
\hline $0,64 \cdot 10^{-6}$ & 11,2 & 28,5 & & 46.4 & $0,64 \cdot 10^{-6}$ \\
\hline $0,95 \cdot 10^{-6}$ & 9,2 & 23,4 & & 38,1 & $0,95 \cdot 10^{-6}$ \\
\hline
\end{tabular}

Subsequent water flow rates are shown in Table 7.

Table 7

Estimated value of water consumption, $\mathrm{kg} / \mathrm{s}$

\begin{tabular}{c|l|l|l|l}
\hline \multirow{2}{*}{$\begin{array}{c}\text { Section, } \\
\mathrm{m}^{2}\end{array}$} & \multicolumn{4}{|c}{ Thickness, $\mathrm{m}$} \\
\cline { 2 - 5 } & 0,0002 & 0,0005 & 0,0008 & 0,001 \\
\hline $0,4 \cdot 10^{-6}$ & 0,006 & 0,014 & 0,023 & 0,03 \\
\hline $0,64 \cdot 10^{-6}$ & 0,007 & 0,02 & 0,03 & 0,04 \\
\hline $0,95 \cdot 10^{-6}$ & 0,009 & 0,02 & 0,04 & 0,045 \\
\hline
\end{tabular}

The required water pressure in the system is determined from the expression

$$
P_{\text {s }}=\frac{Q_{m}^{2}}{2 \cdot \mu^{2} \cdot S^{2} \cdot \rho},
$$

where $\mu=0.62$ (for water) is the flow coefficient for low-viscosity liquids.Subsequent water pressures are summarized in Table 8.

Table 8

Estimated value of water pressure, $\mathrm{Pa}$

\begin{tabular}{c|c|c|c|c}
\hline \multirow{2}{*}{ Section, $\mathrm{m}^{2}$} & \multicolumn{4}{|c}{ Thickness, $\mathrm{m}$} \\
\cline { 2 - 5 } & 0,0002 & 0,0005 & 0,0008 & 0,001 \\
\hline $0,4 \cdot 10^{-6}$ & $0,029 \cdot 10^{6}$ & $0,17 \cdot 10^{6}$ & $0,44 \cdot 10^{6}$ & $0,69 \cdot 10^{6}$ \\
\hline $0,64 \cdot 0^{-6}$ & $0,0163 \cdot 10^{6}$ & $0,105 \cdot 10^{6}$ & $0,28 \cdot 10^{6}$ & $0,44 \cdot 10^{6}$ \\
\hline $0,95 \cdot 10^{6}$ & $0,011 \cdot 10^{6}$ & $0,07 \cdot 10^{6}$ & $0,19 \cdot 10^{6}$ & $0,29 \cdot 10^{6}$ \\
\hline
\end{tabular}

Conclusion. The conducted research led to the following conclusions: 
1. It has been proposed to replace the traditional roll former system with the hydraulic roll former. To test the idea of the hydraulic forming of rolls, a model of a coiler was made.

Research carried out at the Department of Technological Machines, Transport and Logistics at Satbayev University confirmed the efficiency of this proposal and made it possible to make a number of developments and recommendations for creating an industrial coiler.

2. The performed calculations showed the possibility of replacing the mechanical formation with the hydraulic one using a working medium - water and air.

3. In order to study the combined formation in laboratory conditions, a model of a coiler was created, which was structurally tied to the existing laboratory rolling mill "DUO-100".

4. Studies carried out on a laboratory model of a coiler with combined coil formation, have confirmed the fundamental possibility of using the proposed method of coiling for working with various materials.

5. The results of laboratory work indicate a sufficient convergence of calculations with experimental data, which allows using the results obtained as recommendations for the creation of industrial installations.

The use of the combined principle for the formation of the first turns of the roll allows avoiding contact between the rolled metal and the strip, eliminates the appearance of rust due to water residues, expands the adjustment range and reduces the requirements for its accuracy.

\section{References}

1. Grudev A. P., Mashkin L. F., Khanin M. I., (1994). Technology of rolling production: textbook. M.: Metallurgy 
2. Rudskoy A.I., Lunev V.A. (2005). Theory and technology of rolling production: Textbook. - SPb. Nauka

3. Korolev A.A. (1981). Rolling mills and equipment for rolling shops. Atlas: Textbook for universities. -2 nd ed. - M.: Metallurgy

4. Sverdlik G.I., Sobolev S.E. (2013). Technological machines and equipment for metallurgical production. Methodical instructions for practical exercises. Vladikavkaz

5. Yatunchi K, Noma Y, Shimizu R, Tanomoto S., (1982). Unmanned operation technology for a hot strip mill. Jron and Streel Engineer, v. 59, № 11

6. Samways №.1. (1984). Dofasco: Canada’s largest tlat rolled producer. Iron and Steel Engineer, v.61, n 9, p.t.83 -t 101.

7. Juschba R., Stanbermann H. (1985). Hot-strip coiler process technology. - Metallurgical Plant and Technology, n 2, p. 42-47.

8. Ogashi H., Imail I., (1983). New generation hot strip mill at nipon Steel, s Ywata works/ E. Toda e.s.-Iron and Steel engineer, v.60, n 12, p.53-61.

9. Carroll W.P., Macneil P.A. (1965). Design and start-up of the Stelco $2050 \mathrm{~mm}$ hot strip mill. -Iron and Steel Engineer, , v. 62, n 4, p. 39-45.

10. Morennikov, N.V., (1987). Certificate of authorship № 1362528. USSR. The drum of the coiler of the strip rolling mill. Beekeeper. Publ., B.I. №. 48.

11. Davilbekov N.Kh., Kurapov G.G., Pepelyaev I.V., Eskulov S.S., Klement'ev V.A., Mizyurin V.V., Kolynyuk E.P., Elemesov K.K. Method of coiling on a drum of a coiler of a strip mill. Patent RK №. 6363.

12. Patent № 2096873 Great Britain. A device for threading a metal strip into a coiler. Publ. 27.10.82.

13. Davilbekov N.Kh., Kurapov G.G., Elemesov K.K. (1999). Improving the operation of broadband coilers. Proceedings of the International Symposium dedicated to the 100th anniversary of the birth of K.I.Satpayev. Almaty: KazNTU,.S. 268-270.

14. Davilbekov N.Kh., Kurapov G.G., Eskulov S.S, Elemesov K.K., (1995). Device for coilers for coiling strips. Technology of production and processing of ferrous metals. Almaty, RIK on educational and methodological literature, pp. 6164. 